Published in final edited form as:

Obstet Gynecol. 2018 April ; 131(4): 635-641. doi:10.1097/AOG.0000000000002536.

\title{
Simultaneous Compared With Interval Medical Abortion Regimens Where Home Use is Restricted
}

\author{
Patricia A. Lohr, MD, MPH, \\ British Pregnancy Advisory Service \\ Jennifer E. Starling, MSc, \\ Department of Statistics and Data Sciences, The University of Texas at Austin \\ James G. Scott, PhD, and \\ Department of Information, Risk and Operations Management, Department of Statistics and Data \\ Sciences, The University of Texas at Austin
}

Abigail R.A. Aiken, MD, PhD, LBJ

School of Public Affairs and Population Research Center, The University of Texas at Austin

\section{Abstract \\ Objective-To evaluate outcomes with simultaneous administration of mifepristone and misoprostol for medical abortion at $\$ 33$ days of gestation in the year after its implementation in a British clinic system.}

Methods-We conducted a retrospective cohort study using de-identified data from the electronic booking and complications databases and medical records of women who underwent medical abortion at British Pregnancy Advisory Service. Our primary outcome was treatment success with simultaneous dosing versus a regimen with a $24-48$ hour interval between medications. We defined success as complete abortion without surgical evacuation and without continuing pregnancy. To assess relative regimen effectiveness while accounting for selfassignment to simultaneous or interval dosing, we modeled the probability of treatment success using logistic regression with propensity-score adjustment for demographic and clinical characteristics. Secondary outcomes were reasons for abortion failure and clinically significant adverse events (hospital admission, blood transfusion, intravenous antibiotic administration).

\begin{abstract}
Results-Of 28,901 women treated between May 2015 and April 2016, 85\% chose simultaneous dosing. Overall success rates were high with both regimens but lower with simultaneous than with interval dosing (94.5\% vs. $97.1 \%$ respectively, adjusted RR 0.973 , 95\% CI 0.967-0.979). For both regimens, success rates were lower at higher gestational ages, but the relative effectiveness of simultaneous dosing did not vary significantly with gestational age $(\mathrm{p}=0.268)$. Surgical intervention rates for continuing pregnancy were lowest at $\leqslant 49$ days of gestation $(1.4 \%$
\end{abstract}

Corresponding author: Patricia A. Lohr, British Pregnancy Advisory Service, 20 Timothy's Bridge Road, Stratford upon Avon, CV37 9BF UK, patricia.lohr@bpas.org, tel: +44 (0) 3453655050.

Financial Disclosure

Patricia A. Lohr is the Medical Director of British Pregnancy Advisory Service from which the data analyzed are derived. The other authors did not report any potential conflicts of interest.

Each author has indicated that he or she has met the journal's requirements for authorship. 
simultaneous vs. $0.2 \%$ interval, $\mathrm{p}<0.001)$ and highest at $57-63$ days $(5.0 \%$ and $2.2 \%, \mathrm{p}<0.001)$. The rate of clinically significant adverse events was $0.2 \%$ and did not differ by regimen $(\mathrm{p}=0.972)$.

Conclusion-Simultaneous administration of mifepristone and misoprostol is $97 \%$ as effective as a 24-48 hour interval at all gestational ages $\$ 33$ days, with no increase in the risk of clinically significant adverse events. Pragmatic use of simultaneous dosing is reasonable given the small difference in effectiveness.

\section{Introduction}

Home-use of mifepristone and misoprostol for early medical abortion is safe and acceptable, and women prefer it over use in a clinic $(1,2)$. In England and Wales, however, current application of the abortion law does not permit the use of abortion medications outside of registered medical facilities $(3,4)$. The recommended regimen for medical abortion at $\$ 33$ days of gestation in Britain is $200 \mathrm{mg}$ oral mifepristone followed by $800 \mathrm{mcg}$ vaginal misoprostol 24-48 hours later (5). This regimen requires at least two clinic visits, which may be inconvenient and costly, and may negatively impact women's attitudes toward medical abortion $(6,7)$.

To create a more accessible medical-abortion service, British Pregnancy Advisory Service (BPAS) - a non-profit abortion provider with 60 clinics in England and Wales-introduced the option of simultaneous administration of mifepristone and misoprostol in 2015. Prior research shows that simultaneous dosing is effective for early medical abortion, although the rate of side effects may be higher, and the need for more than one dose of misoprostol to achieve a complete abortion may arise more frequently, than with a 24-hour interval between medications $(8,9,10,11,12)$. To avoid a second clinic visit, however, women may still prefer simultaneous dosing where home-use of misoprostol is restricted, despite potential differences between regimens. Our primary objective was to compare effectiveness of the simultaneous regimen to one with a $24-48$ hour interval between medications, both overall and across gestational-age categories. We also compared uptake, reasons for failure, and rates of clinically significant adverse events between regimens.

\section{Materials and Methods}

We conducted a retrospective cohort study using data from early medical abortions provided at BPAS from 1 May 2015-30 April 2016. We obtained data from BPAS' electronic Booking and Invoicing System, which contains the services provided to clients as well as demographic and selected clinical characteristics. These data are initially entered by operators at BPAS' telephone contact center and are validated by clinicians at in-person consultations and at the time of treatment. To link these data with adverse events, client identification numbers were cross-referenced with BPAS' electronic complications database. Complications are identified during follow-up visits, or notified to BPAS by other care providers (e.g., general practitioners, hospitals) or by women themselves. When possible, hospital discharge summaries or letters from general practitioners are obtained to confirm diagnoses and interventions. Complications are recorded on incident forms that are coded using standardized definitions (13) and sent to BPAS Head Office. Codes are checked for accuracy by a clinical lead before entry into the database by a data manager. To ensure 
complete reporting of surgical interventions, staff cross-checked the Booking and Invoicing System for any appointments at BPAS after the date(s) of treatment and hand-checked medical records if a continuing pregnancy, retained non-viable gestational sac or embryo, or incomplete abortion was recorded in the complications database. This study was approved and exempted from full human-subjects review by BPAS' Research and Ethics Committee and the Institutional Review Board at The University of Texas at Austin because all data were pre-existing and provided in a fully de-identified format.

Women with pregnancies of $\$ 33$ days of gestation as determined by abdominal or vaginal ultrasound, who wanted a medical abortion, and who had no contraindications were offered a choice between $200 \mathrm{mg}$ oral mifepristone followed by $800 \mathrm{mcg}$ vaginal misoprostol within 15 minutes (simultaneous administration) or 24-72 hours later. They chose their preferred regimen after being informed of expected differences in side effects and outcomes. Our analytic sample consists of women who chose simultaneous dosing or a 24-48 hour interval between medications.

Two weeks post-treatment, women could choose to return for a vaginal ultrasound, or to use a low-sensitivity urine pregnancy test (detection limit 1000 IU human chorionic gonadotrophin) and symptom checklist to determine the outcome of the abortion themselves (14). Women could schedule a visit at any time if they had concerns or signs and symptoms of a possible complication (e.g., persistent pain, still feeling pregnant). Women diagnosed at a follow-up visit with a retained non-viable sac or embryo or with an incomplete abortion could choose to receive another dose of $800 \mathrm{mcg}$ vaginal misoprostol or have surgical management. Women diagnosed with continuing pregnancies were offered surgical evacuation. Uterine evacuation was also performed at any time if it was clinically necessary, e.g., for hemorrhage, or if a woman requested it.

Our primary outcome was treatment success. We created a binary variable for successful medical abortion $(1=$ success, $0=$ failure). Success was defined as complete expulsion of the uterine contents without surgical intervention and without continuing pregnancy, with reference to the Medical Abortion Reporting of Efficacy Guidelines (15).

In our primary outcome analysis, we compared unadjusted and adjusted rates of successful abortion between regimens. To estimate unadjusted success rates, we calculated binomial confidence intervals for each regimen, both overall and by gestational age group: $\$ 49$ days, 50-56 days, and 57-63 days. We compared the regimens with two-sample tests for a difference in proportions (Appendices 1 and 2, available online at http://links.lww.com/xxx). However, because women self-assigned to regimen, there is the potential for these unadjusted success rates to be confounded by differences in pre-treatment characteristics. We therefore modeled the probability of treatment success using logistic regression with propensity-score adjustment $(16,17,18,19)$. The propensity score represents the estimated conditional probability of assignment to the simultaneous or interval dosing regimen, given client characteristics. Incorporating the propensity score in our model allows us to assess how the probability of successful abortion varies by regimen and gestational age, controlling for observed pre-treatment characteristics that may affect regimen choice. 
To estimate propensity scores, we constructed a random-forest classification model $(16,20)$ using each woman's pre-treatment characteristics as features for predicting her chosen regimen ( $0=$ interval, $1=$ simultaneous). Pre-treatment characteristics included were age in years, Body Mass Index (BMI) in $\mathrm{kg} / \mathrm{m}^{2}$, self-identified race or ethnicity, previous births, abortions, miscarriages and Cesarean sections, and gestational age group on the day of mifepristone administration. The random-forest model allows use of propensity scores without making strong parametric assumptions about the functional form of the relationship between client-level features and treatment assignment. It also offers the added benefit of handling complex interactions among the features. (Appendices 3 and 4, available online at http://links.lww.com/xxx)

Estimated propensity-score quintiles (21) were included as a covariate in our logistic regression models for successful abortion. We first fit an overall model across all gestational ages, using abortion regimen, gestational age, and propensity-score quintile as predictors. We also fit models separately by gestational-age group, using abortion regimen and propensity-score quintile as predictors. We used the method described by Greenland (22) to estimate an adjusted relative risk for the simultaneous regimen from each logistic-regression model. This yielded an overall adjusted relative risk across all gestational ages, as well as an adjusted relative risk specific to each gestational age group. We used Tukey's range test to assess whether the relative risk of success for the simultaneous regimen compared to the interval regimen varied across gestational-age groups. (Appendices 3 and 4, http:// links.lww.com/xxx)

Secondary outcomes were clinically significant adverse events and medical abortion failure. Failure was defined as: 1) the need for surgical uterine evacuation; or 2) continuing pregnancy identified at a follow-up visit, where the women either chose not to complete the abortion or was lost to follow up after diagnosis. Clinically significant adverse events were defined as hospitalization, blood transfusion, and intravenous (IV) antibiotic administration. We compared each adverse event category and a combined "any" category. Women could experience more than one adverse event, but each woman appears only once in the "any" category. All secondary-outcome analyses were conducted using binomial confidence intervals and two-sample tests for a difference in proportion, both overall and separately by gestational-age group. Data analyses were conducted using the R statistical software package version 3.3.3. A p-value of $<0.05$ was considered statistically significant.

\section{Results}

A total of 28,901 women underwent early medical abortion during the 12-month study period. Of these, 24,543 (84.9\%) chose the simultaneous regimen, while 4,358 (15.1\%) chose the 24-48- hour interval regimen. Women who chose the simultaneous regimen were more likely to be older ( $\mathrm{p}=0.028)$; to self-identify as white $(\mathrm{p}<0.001)$; to have had one or more previous births $(\mathrm{p}<0.001)$, abortions $(\mathrm{p}<0.001)$ or miscarriages $(\mathrm{p}<0.001)$; and to be of a lower gestational age $(\mathrm{p}<0.001)$. See Table 1 .

Results for the primary-outcome analysis (successful medical abortion) are detailed in Table 2 , which reports both unadjusted and adjusted rates and relative risks. Statistical adjustment 
using propensity scores made only modest differences to the raw success rates, which reassuringly suggests that confounding due to observed patient characteristics was weak. Nonetheless, we focus here on the adjusted numbers arising from our logistic-regression models (Appendices 5-15, available online at http://links.lww.com/xxx).

The overall success rate was high for both regimens but was slightly lower overall with simultaneous dosing than with interval dosing: $94.5 \%$ vs. $97.1 \%$ respectively (absolute difference of $2.6 \%, \mathrm{p}<0.001)$. Overall, the relative risk of success for simultaneous dosing was 0.973 (95\% CI $0.967-0.979, \mathrm{p}<0.001)$. In this context, "relative risk" is a statistically correct but potentially confusing term, given that our outcome is successful medical abortion. For this reason, the relative risk of 0.973 is best interpreted as "97.3\% relative effectiveness" of the simultaneous regimen versus the interval regimen.

Treatment success rates declined as gestational age increased with both regimens, but the relative risk of success of simultaneous versus interval dosing remained nearly constant within each gestational-age category: $\$ 49$ days, $R R=0.978$ (95\% CI 0.970-0.985); 50-56 days, $R R=0.965$ (95\% CI 0.954-0.977); 57-63 days, RR=0.967 (95\% CI 0.950-0.984). Tukey's range test showed that the relative risk of success of the simultaneous regimen compared to the interval regimen did not vary significantly across gestational-age groups ( $\mathrm{p}$ $=0.268$ ), despite the relatively large sample sizes (Appendix 9, http://links.lww.com/xxx).

Results for the first of our two secondary-outcome analyses (reasons for medical abortion failure) are detailed in Table 3. Continuing pregnancy after treatment was diagnosed in 596 women who chose the simultaneous regimen and 39 who chose the interval regimen: $2.4 \%$ (95\% CI $2.2 \%-2.5 \%$ ) vs. $0.9 \%$ (95\% CI $0.6 \%-1.2 \%$ ) respectively ( $<<0.001)$. Rates of surgical intervention for continuing pregnancy were lowest in both groups at $\$ 49$ days of gestation ( $1.4 \%$ simultaneous vs. $0.2 \%$ interval, $\mathrm{p}<0.001)$, but increased with gestational age and were highest in the 57-63 day group (5.0\% simultaneous vs. $2.2 \%$ interval, $\mathrm{p}<0.001$ ). Eighteen women with continuing pregnancies in the simultaneous group, and one in the interval group, either chose not to complete the abortion or were lost to follow-up after diagnosis. We analyzed surgical evacuation for non-viable retained gestational sac or embryo and incomplete abortion together due to the small number in each category when separated by gestational age group. Surgical evacuation for retained non-viable gestational sac or embryo or for incomplete abortion was more common with the simultaneous regimen overall: $3.1 \%$ simultaneous (95\% CI $2.8 \%-3.3 \%$ ) vs. $2.0 \%$ interval (95\% CI $1.6 \%-2.5 \%$, $\mathrm{p}<0.001)$. When considered separately by gestational age, the difference between regimens was significant at $\$ 49$ days $(2.9 \%$ vs. $1.7 \%, \mathrm{p}=0.003)$ and $50-56$ days $(3.5 \%$ vs. $2.0 \%$, $\mathrm{p}=0.007$ ), but not at $57-63$ days ( $3.1 \%$ vs. $2.6 \%, \mathrm{p}=0.447$ ). Table 3 shows all confidence intervals separated by gestational age. No other indications for surgical uterine evacuation were reported.

Results for our secondary-outcome analysis of clinically significant adverse events are shown in Table 4. Rates of clinically significant adverse events were low with both regimens. Forty-nine of 24,543 women in the simultaneous group $(0.20 \%, 95 \%$ CI $0.15 \%-$ $026 \%)$ versus 8 of 4,358 women in the interval group $(0.18 \%, 95 \%$ CI $0.08 \%-0.36 \%)$, experienced any clinically significant adverse event $(\mathrm{p}=0.972)$. There were no significant 
differences between regimens within each individual adverse-event category. However, there were very few cases of IV antibiotic administration ( 1 event in the simultaneous group, 1 in the interval group) or of blood transfusion ( 8 simultaneous, 4 interval), limiting our power to detect small rate differences within these individual adverse-event categories.

\section{Discussion}

In this retrospective cohort study, we observed that simultaneous administration of mifepristone and misoprostol for medical abortion is $97 \%$ as effective as a regimen with a 24-48 hour interval between medications at all gestational ages up to and including 63 days, even after adjusting for self-selection to regimen. As gestational age increases, absolute success rates decline with both regimens, but the relative effectiveness of the simultaneous regimen remains constant. Moreover, the safety profiles of both regimens are similar, with low rates of clinically significant adverse events. Likely reflecting its greater convenience, women opted overwhelmingly for simultaneous administration.

Two randomized trials have compared outcomes with simultaneous and 24-hour-interval regimens for early medical abortion. Goel et al (11), randomized 80 women to either simultaneous use or a 24-hour interval at $\leq 7$ weeks of gestation and observed success rates of $95.0 \%$ and $97.5 \%$, respectively $(\mathrm{p}=0.56$ ). A randomized non-inferiority study by Creinin and colleagues found that a simultaneous regimen was non-inferior to a 24-hour interval at $\$ 63$ days of gestation. Our overall success rates were similar to those found in both studies, and the significant difference in effectiveness we documented between regimens was within the margin Creinin et al. thought clinically acceptable (10). The Creinin trial found no decrease in treatment success rates with either regimen at higher gestational ages. By contrast, we observed that success rates were lower at higher gestational ages with both regimens. Our findings are consistent with a systematic review of medical abortion trials involving over 45,000 women using $200 \mathrm{mg}$ oral mifepristone and a range of misoprostol regimens and intervals, which found a higher risk of failure (defined as need for surgical evacuation) in groups that had $>25 \%$ of women in the 9th week of pregnancy compared to lower gestations (23). Our study makes two unique contributions by examining regimen preference in a setting where women must return to the clinic to receive misoprostol, and by examining whether there is a change in the relative effectiveness of the two regimens as gestational age advances.

We observed a slightly higher overall rate of clinically significant adverse events than previously reported by Cleland et al. in their evaluation of 233,805 medical abortions performed over two years in Planned Parenthood clinics (24). While our rates of IV antibiotic administration and blood transfusion were similar to those found by Cleland et al., we observed higher rates of hospital admission. This difference may be a function of care in a publically funded health system in Britain rather than an actual difference in the severity of incidents.

One limitation of our study is a lack of information about factors influencing women's choice of regimen. Women received counseling on the expected differences in side effects based on published data and on outcomes from a small pilot of simultaneous administration 
conducted at BPAS. We do not know, however, if or how counseling impacted women's decision-making or experience. Clinicians refer to comparison tables in a printed client guide when informing clients about treatment options and provide women with the guide for future reference, but counseling is not otherwise standardized. A second limitation is that despite efforts to ensure complete reporting of complications, some women may still have received treatment that BPAS clinicians were unable to confirm. Moreover, management of the same adverse event can differ between clinicians and across institutions. Finally, women were not randomized to abortion regimen. We accounted for this limitation using propensity scores, and while the unadjusted and adjusted findings were reassuringly similar, we cannot entirely eliminate the possibility of unobserved confounding factors (Appendices 10 and 11, http://links.lww.com/xxx).

Our findings have several important implications for clinical practice and policy. In settings such as England and Wales, where the use of abortion medications outside of a registered facility is prohibited, interval regimens require at least two clinic trips, which creates substantial access barriers and limits treatment acceptability (25). When given the choice of avoiding multiple clinic visits, women in our sample overwhelmingly opted for simultaneous over interval administration. Our findings also indicate that women can be offered the simultaneous regimen at all gestational ages up to and including 63 days without concern that its relative effectiveness will deteriorate significantly compared with the interval regimen. A $24-48$ hour interval between mifepristone and misoprostol is commonly recommended based on the greatest chance of success (5). However, in light of its potential to better meet the needs of women while still achieving a successful abortion in most cases, medical abortion guidelines should include the option of simultaneous dosing up to and including 63 days of gestation.

\section{Supplementary Material}

Refer to Web version on PubMed Central for supplementary material.

\section{Acknowledgments}

James G. Scott is supported by CAREER grant DMS-1255187 from the U.S. National Science Foundation. Abigail R.A. Aiken is supported by grant P2CHD042849, awarded to the Population Research Center at The University of Texas at Austin by the Eunice Kennedy Shriver National Institute of Child Health and Human Development.

The authors thank Jeanette Taylor, Steve Cheung and Sue Sarson for data acquisition.

\section{References}

1. Gold M, Chong E. If we can do it for misoprostol, why not for mifepristone? The case for taking mifepristone out of the office in medical abortion. Contraception. 2015; 92:194-6. [PubMed: 26093187]

2. Ngo TD, Park MH, Shakur H, Free C. Comparative effectiveness, safety and acceptability of medical abortion at home and in a clinic: a systematic review. Bull World Health Organ. 2011; 89:360-70. [PubMed: 21556304]

3. [Accessed 14/06/2017] Abortion Act 1967 (c. 87). http://www.legislation.gov.uk/ukpga/1967/87/ contents

4. BPAS v Secretary of State for Health [2011] EWHC 235 (Admin). 
5. Royal College of Obstetricians and Gynaecologists. The care of women requesting induced abortion. RCOG; London: 2011.

6. Grossman D, Ellertson C, Grimes DA, Walker D. Routine follow-up visits after first-trimester induced abortion. Obstet Gynecol. 2004; 103:738-45. [PubMed: 15051567]

7. Winikoff B. Acceptability of medical abortion in early pregnancy. Fam Plann Perspect. 1995; 27:142-148. 185. [PubMed: 7589354]

8. Li YT, Chen FM, Chen TH, Li SC, Chen ML, Kuo TC. Concurrent use of mifepristone and misoprostol for early medical abortion. Taiwan J Obstet Gynecol. 2006; 45:325-328. [PubMed: 17175491]

9. Kapp N, Borgatta L, Ellis SC, Stubblefield P. Simultaneous very low dose mifepristone and vaginal misoprostol for medical abortion. Contraception. 2006; 73:525-7. [PubMed: 16627039]

10. Creinin MD, Schreiber CA, Bednarek P, Lintu H, Wagner MS, Meyn LA. Medical Abortion at the Same Time (MAST) Study Trial Group. Mifepristone and misoprostol administered simultaneously versus 24 hours apart for abortion: a randomized controlled trial. Obstet Gynecol. 2007; 109:885-94. [PubMed: 17400850]

11. Goel A, Mittal S, Taneja BK, Singal N, Attri S. Simultaneous administration of mifepristone and misoprostol for early termination of pregnancy: a randomized controlled trial. Arch Gynecol Obstet. 2011; 283:1409-1413. [PubMed: 21431329]

12. Li YT, Hsieh JC, Hou GQ, Chen TH, Chu YC, Lin TC, et al. Simultaneous use of mifepristone and misoprostol for early pregnancy termination. Taiwan J Obstet Gynecol. 2011; 50:11-14. [PubMed: 21482367]

13. BPAS Operational and Clinical Policy and Procedure No. 39, Client Safety Incidents, V5 (April 2015)

14. Cameron ST, Glasier A, Johnstone A, Dewart H, Campbell A. Can women determine the success of early medical termination of pregnancy themselves? Contraception. 2015; 91:6-11. [PubMed: 25300644]

15. Creinin MD, Chen MJ. Medical abortion reporting of efficacy: the MARE guidelines. Contraception. 2016; 94:97-103. [PubMed: 27129936]

16. Austin PC. An Introduction to Propensity Score Methods for Reducing the Effects of Confounding in Observational Studies. Multivariate Behavioral Research. 2011; 46:399-424. [PubMed: 21818162]

17. Austin PC, Mamdani MM. A comparison of propensity score methods: a case-study estimating the effectiveness of post-AMI statin use. Stat Med. 2006; 25:2084-2106. [PubMed: 16220490]

18. Rosenbaum PR. Model-based direct adjustment. The Journal of the American Statistician. 1987a; 82:387-394.

19. Rosenbaum PR, Rubin DB. The central role of the propensity score in observational studies for causal effects. Biometrika. 1983a; 70:41-55.

20. Lee BK, Lessler J, Stuart EA. Improving propensity score weighting using machine learning. Statistics in Medicine. 2010; 29:337-346. [PubMed: 19960510]

21. Rosenbaum PR, Rubin DB. Reducing bias in observational studies using subclassification on the propensity score. Journal of the American Statistical Association. 1984; 79:516-524.

22. Greenland S. Model-based Estimation of Relative Risks and Other Epidemiologic Measures in Studies of Common Outcomes and in Case-Control Studies. American Journal of Epidemiology. 2004; 160:301-5. [PubMed: 15286014]

23. Raymond EG, Shannon C, Weaver MA, Winikoff B. First-trimester medical abortion with mifepristone $200 \mathrm{mg}$ and misoprostol: a systematic review. Contraception. 2013; 87:26-37. [PubMed: 22898359]

24. Cleland K, Creinin MD, Nucatola D, Nshom M, Trussell J. Significant adverse events and outcomes after medical abortion. Obstet Gynecol. 2013; 121:166-71. [PubMed: 23262942]

25. Aiken ARA, Guthrie KA, Schellekens M, Trussell J, Gomperts R. Barriers to accessing abortion services and perspectives on using mifepristone and misoprostol at home in Great Britain. Contraception. 2017 Sep 20. pii:S0010-7824(17)30435-3. [Epub ahead of print]. 


\section{Table 1}

Descriptive characteristics * of women choosing simultaneous or interval administration of mifepristone and misoprostol for early medical abortion from 1 May 2015 to 30 April 2016 at British Pregnancy Advisory Service (N=28,901)

\begin{tabular}{|c|c|c|c|}
\hline Characteristic & Simultaneous $(\mathrm{n}=\mathbf{2 4 , 5 4 3})$ & Interval $(n=4,358)$ & p-value ${ }^{\dagger}$ \\
\hline \multicolumn{4}{|l|}{ Age (Years) } \\
\hline Mean \pm standard deviation & $27.0 \pm 6.6$ & $26.8 \pm 6.7$ & 0.028 \\
\hline Body Mass Index (kg/m²) & & & 0.551 \\
\hline Underweight ( $₫ 8.5$ ) & $7.4(1,821)$ & $7.6(330)$ & \\
\hline Normal (18.5-24.9) & $50.8(12,459)$ & $50.7(2,221)$ & \\
\hline Overweight (25.0-29.9) & $25.6(6,285)$ & $24.8(1,081)$ & \\
\hline Obese ( 230.0 ) & $16.2(3,978)$ & $16.9(736)$ & \\
\hline Race or Ethnicity & & & $<0.001$ \\
\hline White & $77.9(19,115)$ & $74.0(3,227)$ & \\
\hline Asian & $8.6(2,100)$ & $11.7(510)$ & \\
\hline Black & $6.2(1,532)$ & $7.1(310)$ & \\
\hline Other & $5.4(1,322)$ & $5.7(247)$ & \\
\hline Not Reported & $1.9(474)$ & $1.5(64)$ & \\
\hline Previous Abortions & & & $<0.001$ \\
\hline 0 & $62.8(15,407)$ & $67.8(2,953)$ & \\
\hline 1 or more & $37.2(9,136)$ & $32.2(1,405)$ & \\
\hline Previous Births & & & $<0.001$ \\
\hline 0 & $47.0(11,526)$ & $53.5(2,330)$ & \\
\hline 1 or more & $53.0(13,017)$ & $46.5(2,028)$ & \\
\hline Previous Cesarean Sections & & & 0.045 \\
\hline 0 & $88.3(21,680)$ & $89.4(3,896)$ & \\
\hline 1 or more & $11.7(2,863)$ & $10.6(462)$ & \\
\hline Previous Miscarriages & & & $<0.001$ \\
\hline 0 & $82.2(20,179)$ & $84.7(3,691)$ & \\
\hline 1 or more & $17.8(4,364)$ & $15.3(667)$ & \\
\hline Gestational Age (Days) & & & $<0.001$ \\
\hline$\leq 49$ & $57.1(14,016)$ & $46.0(2,005)$ & \\
\hline $50-56$ & $25.8(6,321)$ & $30.2(1,316)$ & \\
\hline $57-63$ & $17.1(4,206)$ & $23.8(1,037)$ & \\
\hline
\end{tabular}




\begin{tabular}{lccc}
\hline Characteristic & Simultaneous $(\mathbf{n}=\mathbf{2 4 , 5 4 3 )}$ & Interval $(\mathbf{n}=\mathbf{4 , 3 5 8})$ & p-value $^{\dagger}$ \\
\hline Median $\pm \mathrm{IQR}$ & $48 \pm 11$ & $50 \pm 11$ & $<0.001$ \\
\hline
\end{tabular}

Results presented as \% (n) unless noted.

Excluding means and standard deviations, p-values are for chi-squared tests for characteristics; null hypothesis is that the joint distribution of the counts in the contingency tables is the product of row and column marginal distributions. For means and standard deviations, $\mathrm{p}$-values are for t-tests to compare treatment versus control group means; null hypothesis is that groups have equal means. For gestational age, the p-value is for a MannWhitney test. 


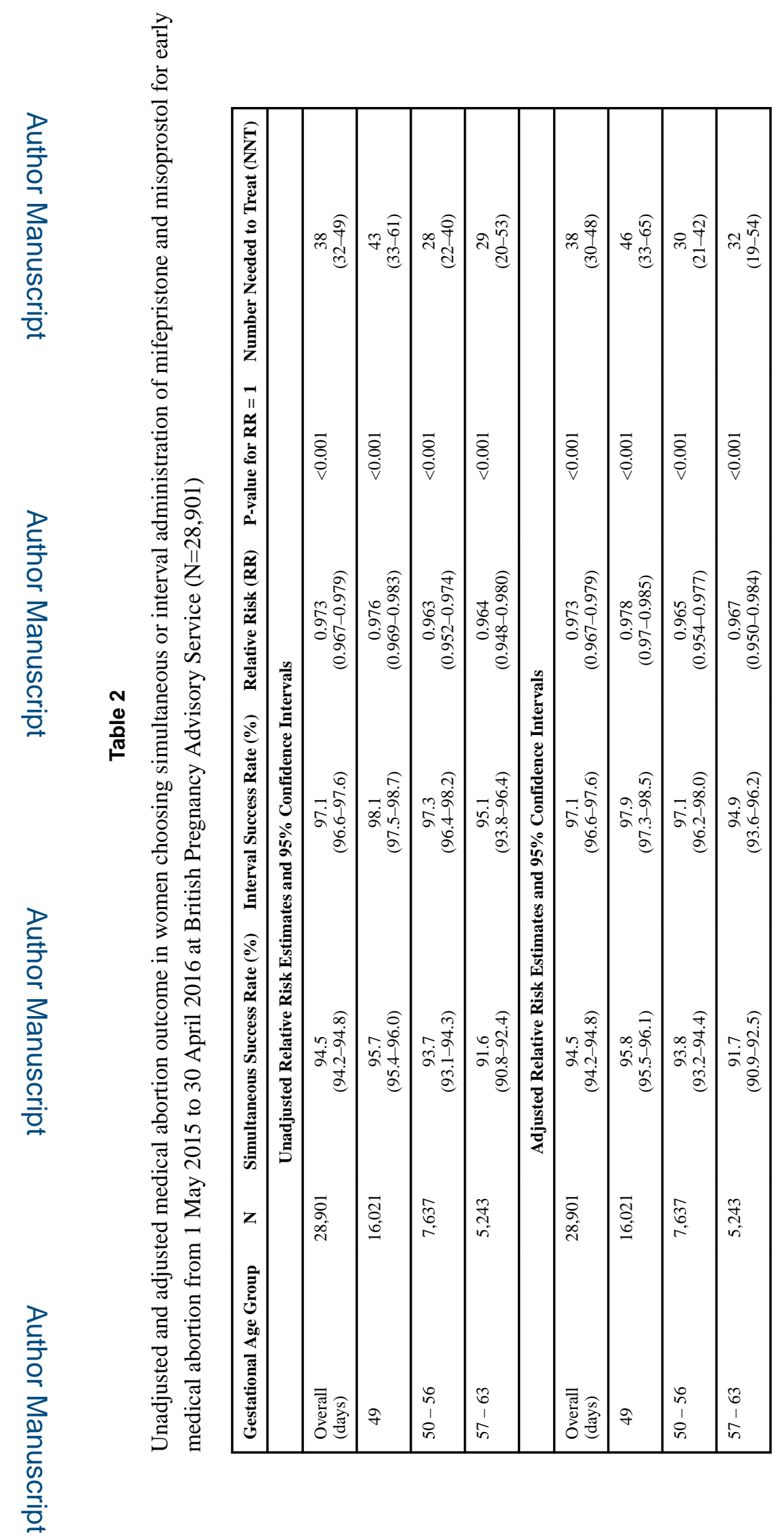

Obstet Gynecol. Author manuscript; available in PMC 2019 April 01. 


\section{Table 3}

Reasons for medical abortion failure among women choosing simultaneous or interval administration of mifepristone and misoprostol for early medical abortion from 1 May 2015 to 30 April 2016 at British Pregnancy Advisory Service $(\mathrm{N}=28,901)$

\begin{tabular}{|c|c|c|c|}
\hline & Simultaneous $(\mathrm{n}=\mathbf{2 4 , 5 4 3})$ & Interval $(n=4,358)$ & p-value \\
\hline & (n) & (n) & \\
\hline Continuing pregnancy (total) & (596) & (39) & \\
\hline Opted against surgical intervention & (18) & (1) & \\
\hline Indications for surgical evacuation & $\begin{array}{c}\%[95 \% \mathrm{CI}] \\
(\mathrm{n})\end{array}$ & $\begin{array}{c}\%[95 \% \mathrm{CI}] \\
(\mathrm{n})\end{array}$ & \\
\hline \multicolumn{4}{|l|}{ Continuing pregnancy } \\
\hline Overall (days) & $\begin{array}{c}2.4[2.2-2.5] \\
(578)\end{array}$ & $\begin{array}{c}0.9[0.6-1.2] \\
(38)\end{array}$ & $<0.001$ \\
\hline$\leq 49$ & $\begin{array}{c}1.4[1.2-1.6] \\
(192)\end{array}$ & $\begin{array}{c}0.2[0.1-0.6] \\
(5)\end{array}$ & $<0.001$ \\
\hline $50-56$ & $\begin{array}{c}2.8[2.4-3.2] \\
(174)\end{array}$ & $\begin{array}{c}0.8[0.4-1.4] \\
(10)\end{array}$ & $<0.001$ \\
\hline $57-63$ & $\begin{array}{l}5.0\left[\begin{array}{l}{[4.4-5.8]} \\
(212)\end{array}\right.\end{array}$ & $\begin{array}{c}2.2[1.4-3.3] \\
(23)\end{array}$ & $<0.001$ \\
\hline \multicolumn{4}{|c|}{ Retained non-viable gestational sac or incomplete abortion } \\
\hline Overall (days) & $\begin{array}{c}3.1[2.8-3.3] \\
(752)\end{array}$ & $\begin{array}{c}2.0[1.6-2.5] \\
(87)\end{array}$ & $<0.001$ \\
\hline$\leq 49$ & $\begin{array}{c}2.9[2.6-3.2] \\
\quad(403)\end{array}$ & $\begin{array}{c}1.7[1.2-2.4] \\
(34)\end{array}$ & 0.003 \\
\hline $50-56$ & $\begin{array}{l}3.5[3.0-3.9] \\
(218)\end{array}$ & $\begin{array}{c}2.0[1.3-2.9] \\
(26)\end{array}$ & 0.007 \\
\hline $57-63$ & $\begin{array}{c}3.1[2.6-3.7] \\
(131)\end{array}$ & $\begin{array}{c}2.6[1.7-3.8] \\
(23)\end{array}$ & 0.447 \\
\hline
\end{tabular}




\section{Table 4}

Clinically significant adverse events* associated with early medical abortion with simultaneous or interval administration of mifepristone and misoprostol from 1 May 2015 to 30 April 2016 at British Pregnancy Advisory Service $(\mathrm{N}=28,901)$

\begin{tabular}{|c|c|c|c|}
\hline Clinically Significant Adverse Event & Simultaneous $(n=24,543)$ & Interval $(n=4,358)$ & p-value \\
\hline & $\begin{array}{c}\%[95 \% \mathrm{CI}] \\
(\mathbf{n})\end{array}$ & $\begin{array}{c}\%[95 \% \mathrm{CI}] \\
(\mathbf{n})\end{array}$ & \\
\hline Any & $\begin{array}{c}0.20[0.15-0.26] \\
(49)\end{array}$ & $\begin{array}{c}0.18[0.08-0.36] \\
(8)\end{array}$ & 0.972 \\
\hline IV antibiotics & $\begin{array}{c}0.000[0.00-0.02] \\
(1)\end{array}$ & $\begin{array}{c}0.02[0.00-0.13] \\
(1)\end{array}$ & 0.695 \\
\hline Blood transfusion & $\begin{array}{c}0.03[0.01-0.06] \\
(8)\end{array}$ & $\begin{array}{c}0.09[0.03-0.23] \\
(4)\end{array}$ & 0.173 \\
\hline Hospital admission & $\begin{array}{c}0.20[0.14-0.26] \\
(48)\end{array}$ & $\begin{array}{c}0.16[0.06-0.33] \\
(7)\end{array}$ & 0.765 \\
\hline
\end{tabular}

* An individual woman may experience more than one significant adverse event. Each woman is counted only once in the 'Any' category, which represents a combined variable for each of the three categories: IV antibiotics, blood transfusion, and hospital admission. 